6 Anderson DJ, Moehring RW, Weber DJ, et al. Effectiveness of targeted enhanced terminal room disinfection on hospital-wide acquisition and infection with multidrug-resistant organisms and Clostridium difficile: a secondary analysis of a multicentre cluster randomised controlled trial with crossover design (BETR Disinfection). Lancet Infect Dis 2018; 18: 845-53

7 Donskey CJ. Does improving surface cleaning and disinfection reduce health care-associated infections? Am J Infect Control 2013; 41: S12-19.

8 Wagenvoort JH, De Brauwer El, Penders RJ, Willems RJ, Top J, Bonten MJ. Environmental survival of vancomycin-resistant Enterococcus faecium. J Hospital Infect 2011; 77: 282-83.
9 Eyre DW, Cule ML, Wilson DJ, et al. Diverse sources of C. difficile infection identified on whole-genome sequencing. NEJM 2013; 369: 1195-205

10 Salgado CD, Sepkowitz KA, John JF, et al. Copper surfaces reduce the rate of healthcare-acquired infections in the intensive care unit. Infect Control Hosp Epidemiol 2013; 34: 479-86.

11 Lawley TD, Clare S, Deakin LJ, et al. Use of purified Clostridium difficile spores to facilitate evaluation of health care disinfection regimens. Appl Environ Microbiol 2010; 76: 6895-900.

\title{
How fluoroquinolones poison the neighbourhood
}

The global crisis in antibiotic resistance results from a complex interplay of multiple variables, all interacting, and, thereby creating unpredictable behaviour. The increasing incidence of infections caused by antibioticresistant bacteria in humans in many parts of the world is, amongst other things, influenced by selective antibiotic pressure and cross-transmission in healthcare settings, transfer of patients and healthy carriers of such bacteria between health-care settings and across the globe, antibiotic-induced selection of resistant bacteria in animals with spillover to humans through direct contact or food, and environmental contamination through human and animal excretions.

By contrast with these well known and widely studied risks, transmission of resistance between humans outside health-care settings in the so-called open population, is less well studied. In this issue of the journal, Marcelo Low and co-workers make an important contribution to elucidating and quantifying this aspect in the epidemiology of antibiotic resistance ${ }^{1}$. They used detailed information in 1733 geographical areas in Israel on healthcare consumption (including antibiotic use) from more than 3 million inhabitants and more than 2 million urine culture results in a sophisticated population-based case-control study. In 300105 events with Escherichia coli growth and 1899168 urine cultures with no growth, they showednot unexpectedly-a dose-response relationship between the amount of fluoroquinolone consumption and the risk of fluoroquinolone-resistant (FQ-R) $E$ coli in urine cultures. The novelty is in the finding that the amount of fluoroquinolone use in the neighbourhood was also associated-again in a dose-dependent manner-with the risk of FQ-R E coli among patients who had not used fluoroquinolones in the year before.
Not surprisingly, the direct association between fluoroquinolone use and FQ-R E coli bacteriuria was stronger than the indirect association in the neighbourhood. When estimating the relative contribution of both mechanisms to all FQ-R E coli, personal fluoroquinolone consumption among female patients accounted for $46 \%$, whereas the neighbourhood fluoroquinolone consumption accounted for about $25 \%$ of the cases. Consequently, $71 \%$ of the FQ-R E coli bacteriuria was explained by direct or indirect exposure to fluoroquinolones, and the direct to indirect contribution was close to a 2:1 ratio. Of note, if the effects of fluoroquinolone use in an individual were to last more than 1 year, then some indirect exposure might in fact have been direct exposure.

Obviously, a higher risk of urinary tract infection with FQ-R E coli in the open population poses a serious threat. Yet, fluoroquinolone consumption was also associatedagain in a dose-dependent manner-with a higher prevalence of culture negative urine samples. This raises the question of whether the associations derived in a retrospective study were subject to observation bias. As it is unlikely that neighbourhood fluoroquinolone consumption sterilises urine in patients who had not used these antibiotics, lower culture positivity prevalence in such patients must result from one or a combination of two possible mechanisms. Possibly general practitioners in neighbourhoods with higher fluoroquinolone consumption had a lower threshold for ordering urine cultures, possibly resulting in more sterile urine cultures, but this would not threaten the validity of the study as the authors restricted the primary analysis to culture positive patients. Furthermore, there might have been differences in the timing of urine cultures between neighbourhoods. If more patients received treatment with fluoroquinolones
@ (1)

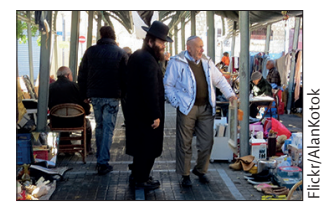

Published Online March 4, 2019 http://dx.doi.org/10.1016/ S1473-3099(18)30737-0

See Articles page 419

This online publication has been corrected.

The corrected version first appeared at thelancet.

com/infection on June 10, 2019 
for urinary tract infections and urine cultures were only obtained when treatment failed, this would have increased the probability of detecting FQ- $R$ E coli relative to fluoroquinolone susceptible (FQ-S) E coli. It is unknown (but would not be surprising) if diagnostic and treatment behaviour were correlated. This second mechanism might endanger the study validity, as acknowledged by the authors. As FQ-R E coli infections are largely outnumbered by susceptible ones, a small decrease in such infections could explain the relatively large increase in FQ-R E coli. If so, this would also have led to overestimation of the indirect effects. It would, therefore, be interesting to compare timing of urine culture collection with antibiotic treatment initiation between neighbourhoods to test this hypothesis.

Finally, as with many other infectious diseases, the results of this study appear to be driven by a subpopulation with substantially higher fluoro-quinolone use than the population average. If we assume that the mean duration of a fluoroquinolone treatment course is 5 days, the mean of 1.5 defined daily dose per 1000 days in the population implies that at most, $11 \%$ of the population received fluoroquinolones in the last year. Reported fluoroquinolone use was $20 \%$ among patients with FQ-S E coli, 26\% among patients with sterile cultures, and $58 \%$ among patients with FQ-R E coli. Consequently, fluoroquinolone use was at least twice the average among patients in which urine samples were tested.

This study emphasises both the power of using large datasets for population-based studies and the consequences of using fluoroquinolones. The observed association between population density and the occurrence of FQ-R E coli suggests that direct transmission between humans has contributed to the findings. This has been shown before for $E$ coli producing extendedspectrum $\beta$-lactamases in Dutch household settings. ${ }^{2,3}$ In both studies, the estimated basic reproductive number was, despite documented transmission, far below one, suggesting that further transmission within, and certainly outside, the household setting is unlikely. The observed two to one ratio between direct and indirect acquisition of FQ-R E coli in Israel suggests that, on average, direct acquisition through antibiotic use does not lead to many indirect acquisitions, suggesting that transmission in the open population will not lead to epidemic spread of these bacteria. The results of the study from Low and coworkers hopefully inspire others to do detailed population-based studies to quantify transmission of antibiotic resistant bacteria to better understand this potentially important but still largely unknown, element in the complex system of antibiotic resistance epidemiology.

Cornelis H van Werkhoven, Martin Bootsma, *Marc Bonten Julius Centre for Health Sciences and Primary Care (CHvW, MBoo, MBon) and Department of Medical Microbiology (MBon), University Medical Centre Utrecht, Utrecht University, the Netherlands; and Mathematical Institute, Utrecht University, the Netherlands (MBoo)

mbonten@umcutrecht.nl

We declare no competing interests.

1 Low M, Neuberger A, Hooton TM, et al. Association between urinary community-acquired fluoroquinolone-resistant Escherichia coli and neighbourhood antibiotic consumption: a population-based case-control study. Lancet Infect Dis 2019; published online March 4. http://dx.doi. org/10.1016/S1473-3099(18)30676-5.

2 Haverkate MR, Platteel TN, Fluit AC, et al. Quantifying within-household transmission of extended-spectrum $\beta$-lactamase-producing bacteria. Clin Microbiol Infect 2017; 23: 46e1-e7.

3 Arcilla MS, van Hattem JM, Haverkate MR, et al. Import and spread of extended-spectrum $\beta$-lactamase-producing Enterobacteriaceae by international travellers (COMBAT study): a prospective, multicentre cohort study. Lancet Infect Dis 2017; 17: 78-85.

\section{(4) Oa The genesis of the Ebola virus outbreak in west Africa}

Published Online

February 21, 2019 http://dx.doi.org/10.1016/ S1473-3099(19)30055-6 This online publication has been corrected. The corrected version first appeared at thelancet.com/infection on March 29, 2019 See Articles page 429
The 2013-16 Ebola virus outbreak in west Africa was purported to have begun in the Guinean village of Meliandou in December, 2013. ${ }^{1}$ Authorities recorded 11 cases of Ebola virus disease (EVD) at this "index site" (where the virus is believed to have first spilled over into the human population), with $100 \%$ case fatality. In The Lancet Infectious Diseases, Joseph WS Timothy and colleagues $^{2}$ present a brilliant piece of epidemiological sleuthing. By combining classic field investigations with an assay that can measure Ebola virus antibodies in oral fluid, the authors have improved our understanding of the early development of the outbreak in Meliandou. They show that there was almost double the number of individuals infected with Ebola virus (21 cases vs the 11 cases previously reported), and the case fatality was $55.6 \%$.

The non-invasive assay used in this study reportedly has high specificity, ${ }^{3}$ although the scarce number 